\title{
Study of the Flux and Spectral Variations in the VHE Emission from the Blazar Markarian 501, with the MAGIC Telescope
}

\author{
D.Paneque* and on behalf of the MAGIC collaboration ${ }^{\dagger}$ \\ ${ }^{*}$ Stanford Linear Accelerator Center (SLAC) / Max-Planck-Institut für Physik, München (MPI) \\ $\dagger$ updated author list can be found at http://wwwmagic.mppmu.mpg.de/col laboration/members
}

\begin{abstract}
The blazar Markarian 501 (Mrk 501) was observed above $100 \mathrm{GeV}$ with the MAGIC Telescope during May, June and July 2005. The high sensitivity of the instrument made possible the detection of the source with high significance in each of the observing nights. During this observational campaign, the emitted gamma-ray flux from Mkn 501 was found to vary by one order of magnitude, and showed a high correlation with spectral changes. Intra-night flux variability was also observed, with flux-doubling times of $\sim 2$ minutes. The data showed a clear evidence of a spectral peak (in the $v F v$ representation) during the nights when the gamma-ray activity was highest. The location of this spectral feature was found to be correlated with the emitted gamma-ray flux. In these proceedings we discuss some of the results of this unprecedented spectral and temporal analysis of Mrk 501 observations in the very high energy range.
\end{abstract}

Keywords: MAGIC, IACT, Mrk 501, Blazar, AGN, $\gamma$-ray astronomy

PACS: $95.85 . \mathrm{Pw}$, 98.70.Rz, 95.55.Ka

\section{OBSERVATION AND RESULTS}

The observations of Mrk 501 in the Very High Energy (VHE) domain were carried out with the Major Atmospheric Gamma-ray Imaging Cherenkov (MAGIC) Telescope (see [2, 7, 3, 4] for details of MAGIC). The net observation time on the source is 31.6 hours (24 nights) between May and July 2005. Quasi-simultaneously to the MAGIC data, Mrk 501 was regularly observed with KVA as a part of Tuorla Observatory's blazar monitoring program ${ }^{1}$. And we also used data taken with the RXTE satellite's All-Sky-Monitor (RXTE/ASM) ${ }^{2}$.

The overall Light Curve (LC) of Mrk 501 during the MAGIC observation campaign is shown in Fig. 1. The observed flux is shown in three energy bands: VHE $(0.15 \mathrm{GeV}-10 \mathrm{TeV}), \mathrm{X}$-rays $(2 \mathrm{keV}-10 \mathrm{keV})$, and optical $(1.5 \mathrm{eV}-$ $2.5 \mathrm{eV}$ ) as measured by MAGIC, RXTE/ASM and KVA, respectively. The X-ray and optical fluxes are computed as weighted averages using RXTE/ASM and KVA measurements taken simultaneously with the MAGIC observations plus/minus a time tolerance of 0.2 days. A smaller time tolerance substantially decreases the number of X-ray points that can be used. The $\gamma$-ray flux level of the Crab Nebula (lilac-dashed horizontal line in the top plot) is also shown in the upper plot of Fig. 1 for comparison. The Crab nebula flux was obtained by applying the very same analysis used for Mrk 501 to the MAGIC Crab nebula data taken during December 2005 under observing conditions similar to those for Mrk 501. The estimated Crab Nebula flux level is therefore roughly affected by the same systematics as the fluxes obtained for Mrk 501. We found $F_{\mathrm{Crab}}(>0.15 \mathrm{TeV})=(3.2 \pm 0.1) \times 10^{-10} \mathrm{~cm}^{-2} \mathrm{~s}^{-1}$, thereafter referred to as Crab Unit (c.u.). Fig. 1 shows that the VHE flux from Mrk 501 was about 0.5 c.u. during most of the observation nights; yet there are significant deviations from this mean $\gamma$-ray emission, with large flux variations occurring in consecutive nights.

During the two nights with the highest VHE activity (>3c.u.), namely June 30 and July 9, Mrk 501 clearly showed intra-night flux variations. The corresponding $\mathrm{LC}$ in the $0.15-10 \mathrm{TeV}$ band is shown in Fig. 2 with a time binning of $\sim 2$ minutes. A constant line fit to the whole LC gives a $\chi^{2} / N D F=47.9 / 30$ (probability $p=2.0 \times 10^{-2}$ ) for the night of June 30 , and a $\chi^{2} / N D F=80.6 / 21\left(p=6.4 \cdot 10^{-9}\right)$ for the night of July 9 . Therefore, the emission above $150 \mathrm{GeV}$ during the two nights is statistically inconsistent with being constant. The burst's amplitude and duration, as well as its rise/fall times, were quantified according to the following function [8]:

\footnotetext{
1 http://users.utu.fi/kani/1m/.

2 The data are publicly available at http://heasarc.gsfc.nasa.gov/xte_weather/.
} 


$$
F(t)=a+\frac{b}{2^{-\frac{t-t_{0}}{c}}+2^{\frac{t-t_{0}}{d}}}
$$

This model parametrizes a flux variation (flare) superposed on a stable emission: $F(t)$ asymptotically tends to $a$ when $t \rightarrow \pm \infty$. The parameter $a$ is the assumed constant flux at the time of the flare (cf. the horizontal black dashed lines in Fig. 2); $t_{0}$ is set to the time corresponding to the point with the highest value in the LC; and $b, c, d$ are left free to vary. The latter two parameters denote the flux-doubling rise and fall times, respectively. The resulting fit parameters are reported in the insets of Fig. 2, showing flux-doubling times of the order of $\mathbf{2}$ minutes. This is the fastest flux variability ever observed from Mrk 501. These bursts were also studied in four non-overlapping energy ranges; 0.15 $0.25 \mathrm{TeV}, 0.25-0.6 \mathrm{TeV}, 0.6-1.2 \mathrm{TeV}$ and $>1.2 \mathrm{TeV}$. The main outcome of this study is a) the burst from June 30 is significant only in the energy range $0.25-0.6 \mathrm{TeV}$, while the burst from July 9 is significantly observed in all energy ranges; $b$ ) in the July 9 burst, the highest energies are delayed by $4 \pm 1$ minutes with respect to the lowest energies; and $c$ ) in the July 9 burst, the relative amplitude of the flux variation increases with energy. Further details from these studies can be found elsewhere [1].

Mrk 501 showed energy-dependent flux variations throughout the entire MAGIC observational campaign. We followed the prescription given in [9] to quantify the flux variability by means of the fractional variability parameter $F_{v a r}$, as a function of energy. The outcome of this calculation is shown in the left-hand plot of Fig. 3; the flux variability increases with energy. The same tendency was reported at X-ray frequencies, but with lower values of $F_{v a r}$ [5].

During our observations, the VHE emission of Mrk 501 was very dynamic, showing significant spectral variability on a timescale of days. Nevertheless, most of the data are well described by a simple Power-law (PL) function. This does not hold for the two flaring nights of June 30 and July 9 which show clear spectral curvature and thus they are fit with a log-parabolic (LP) function.

$$
P L: \frac{d F}{d E}=K_{0} \cdot\left(\frac{E}{0.3 \mathrm{TeV}}\right)^{-a} \quad L P: \frac{d F}{d E}=K_{0} \cdot\left(\frac{E}{0.3 T e V}\right)^{-a-b \cdot \log _{10}\left(\frac{E}{0.3 T e V}\right)}
$$

Here, $K_{0}$ is a normalization factor, $a$ is the spectral index at $0.3 \mathrm{TeV}$, and $b$ is a curvature parameter.

The right-hand plot of Fig. 3 shows the energy spectra (EBL corrected) of Mrk 501 for the flaring nights (June 30 and July 9) for three different flux levels, low, medium and high, which contain 12, 8 and 2 ("non-flaring") nights respectively. See caption of Fig. 3 for definition of flux levels. The data shows very clearly a hardening of the spectra with increasing flux. Besides, the data from the flaring nights suggest the presence of a spectral peak. The location of this peak, determined using the fit parameters and errors from the log-parabolic fit, is $0.85 \pm 0.13 \mathrm{TeV}$ and 0.44 $\pm 0.08 \mathrm{TeV}$ for June 30 and July 9 , respectively. If this peak exists for the data sets low, medium and high, then its location is certainly below $0.1 \mathrm{TeV}$. Therefore, the location of such spectral peak is very probably correlated with the source luminosity.

\section{CONCLUSIONS}

We have undertaken a systematic study of the temporal and spectral variability of the nearby blazar Mrk 501 with the MAGIC telescope at energies $>0.1 \mathrm{TeV}$. During 24 observing nights between May and July 2005, all of which yielded significant detections, we measured fluxes and spectra at levels of baseline activity ranging from $<0.5$ to $>1$ c.u.. During two nights, on June 30 and July 9, Mrk 501 underwent into a very active state with a $\gamma$-ray emission $>3$ c.u., and flux-doubling times of $\sim 2$ minutes. An overall trend of harder spectra for higher flux was clearly seen. The VHE $\gamma$-ray variability was found to increase with energy, and it is significantly higher than the variability at X-ray frequencies. A spectral peak, at a location dependent on source luminosity, was clearly observed during the active states. All these features are naturally expected in synchro-self-Compton (SSC) models of blazar VHE emission. See [1] for further details from these observations. 

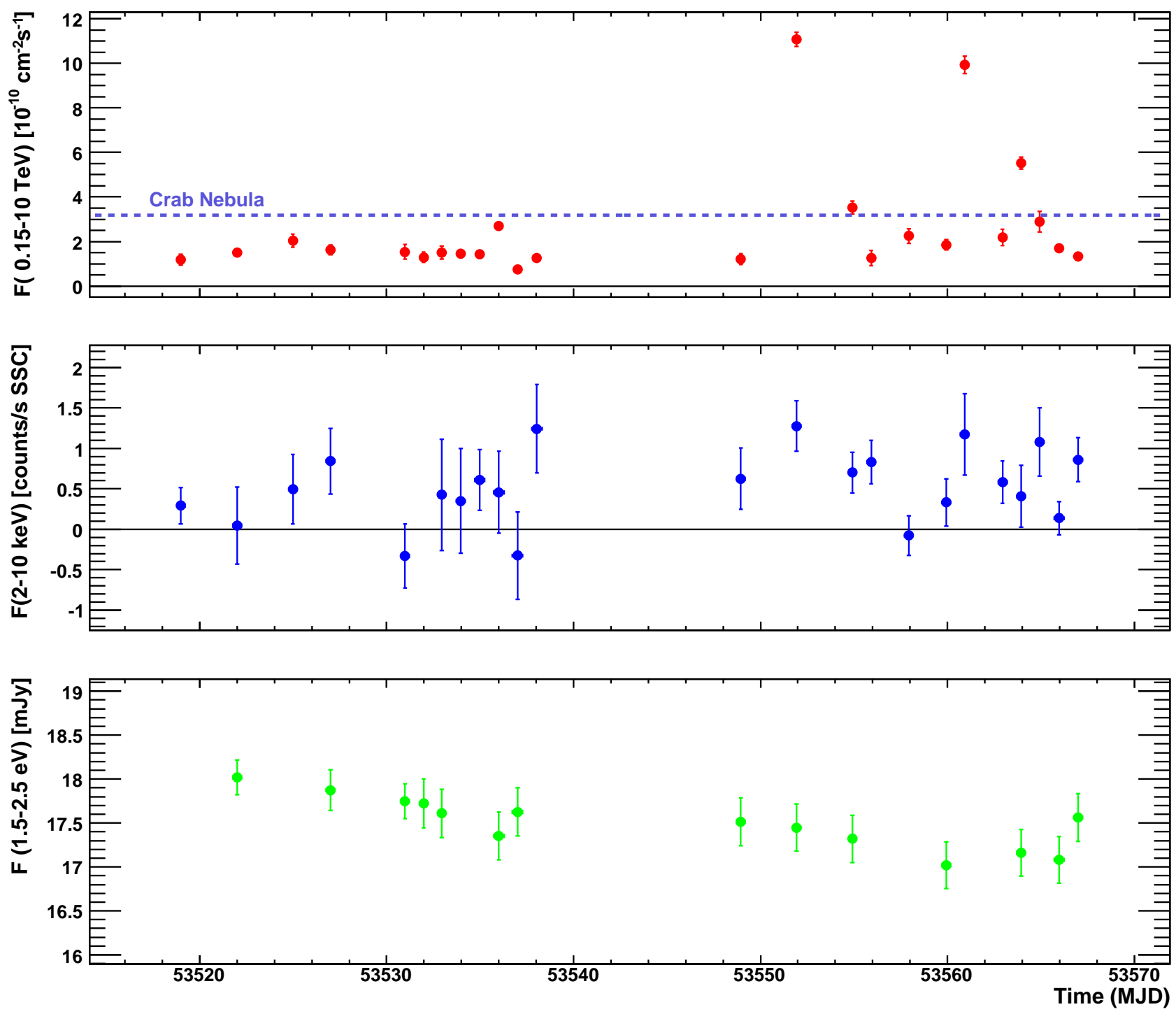

FIGURE 1. Multi-frequency LC during the MAGIC observations of Mrk 501 (May-July 2005). Top) MAGIC flux above 0.15 $\mathrm{TeV}$. The Crab flux is also shown for comparison (lilac dashed horizontal line). Middle) RXTE/ASM 2-10 keV flux. Bottom) KVA $\sim 1.5-2.5 \mathrm{eV}$ flux. Error bars denote $1 \sigma$ statistical uncertainties. The X-ray/optical data were selected to match the MAGIC data within a time window of 0.2 days.

\section{REFERENCES}

1. Albert,J et al 2007, Submitted to ApJ, astro-ph/0702008

2. Baixeras, C. et al 2004, Nucl. Instr. Meth. A, 518, 188

3. Cortina, J. et al 2005, in Proc. 29th International Cosmic Ray Conference, Pune (India), astro-ph/0508274

4. Gaug M. 2006, PhD Thesis, IFAE Barcelona http://wwwmagic.mppmu.mpg.de/publications/theses/MGaug.pdf

5. Gliozzi, M. et al 2006, ApJ, 646, 61

6. Kneiske, T. M., et al 2004, A\&A, 413, 807

7. Paneque, D., 2004, PhD Thesis, MPI Munich http://wwwmagic.mppmu.mpg.de/publications/theses/David_thesis.ps.gz

8. Schweizer T. 2004, PhD Thesis, IFAE Barcelona http://wwwmagic.mppmu.mpg.de/publications/theses/ThomasS_thesis.ps.gz

9. Vaughan, S., et al 2003, MNRAS, 345, 1271 

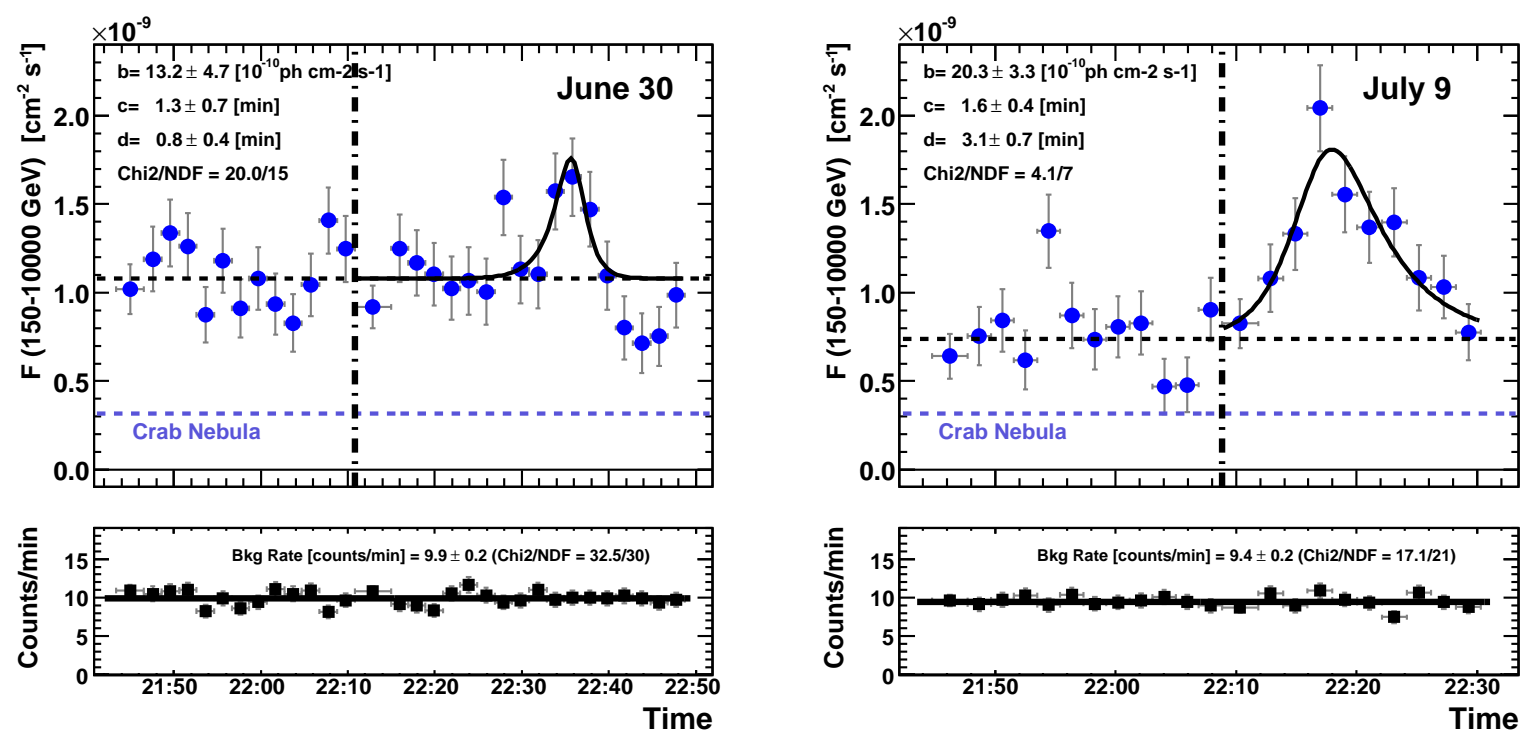

FIGURE 2. Integrated-flux LCs of Mrk 501 for the flare nights of June 30 and July 9. Horizontal bars represent the 2-minute time bins, and vertical bars denote $1 \sigma$ statistical uncertainties. For comparison, the Crab emission is also shown as a lilac dashed horizontal line. The vertical dot-dashed line divides the data into 'stable' (i.e., pre-burst) and 'variable' (i.e., in-burst) emission emission. The horizontal black dashed line represents the average of the 'stable' emission. The solid black curve represents the best-fit flare model (see eq. 1). The insets report the fit parameters and goodness of the fit. The bottom plots show the mean background rate during each of the 2-minute bins of the LCs. The insets report the mean background rate during the entire night, resulting from a constant fit to the data points. The goodness of such fit is also given. The background rates are constant along the entire night. Consequently, the variations seen in the upper panels of the middle and right-hand plot correspond to actual variations of the VHE $\gamma$-ray flux from Mrk501, thus ruling out detector instabilities and/or atmospheric changes.
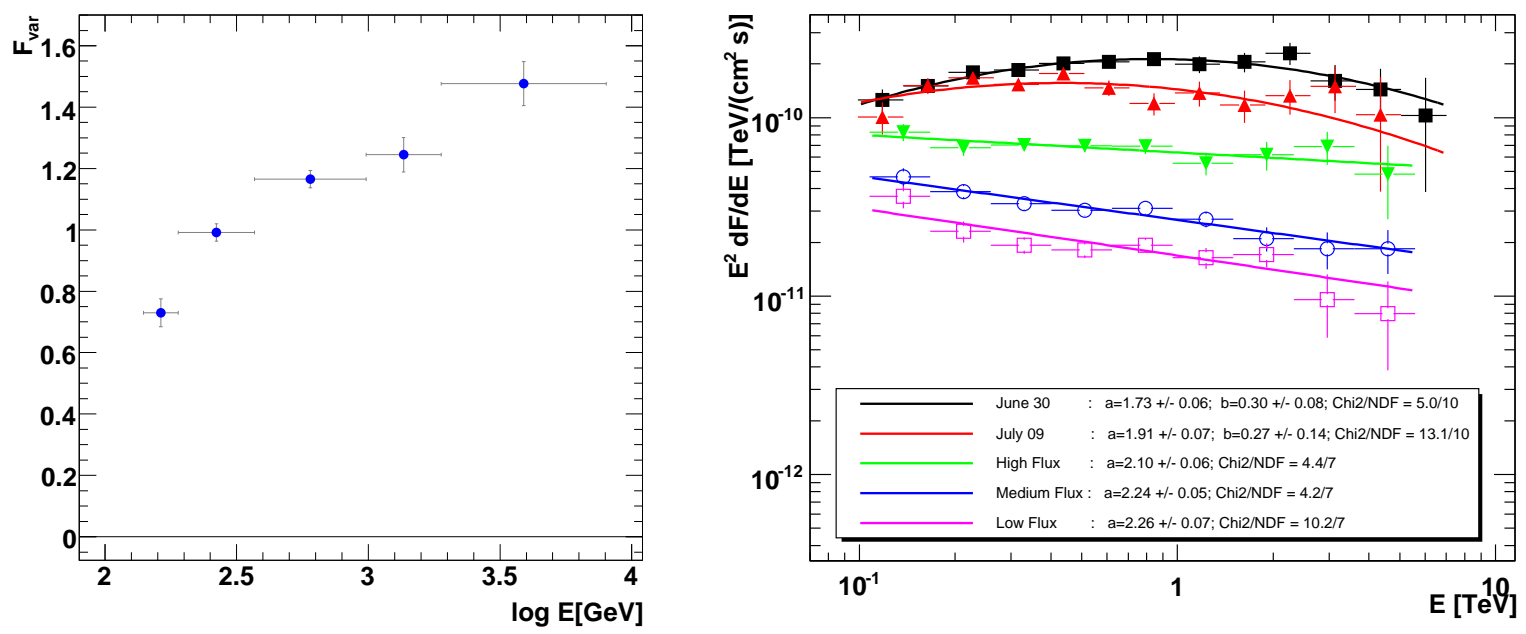

FIGURE 3. Left-hand: Fractional variability parameter, calculated as described in [9]. Vertical bars denote $1 \sigma$ uncertainties, horizontal bars indicate the width of each energy bin. Right-hand: Energy spectra of Mrk 501 for the flaring nights of June 30 (black squares) and July 9 (red up-triangles), and for three data sets which group nights according to whether their integral flux above $150 \mathrm{GeV}, F_{150 \mathrm{GeV}}$ (measured in Crab Units (c.u.)), was high (1.0 c.u. $<F_{150 \mathrm{GeV}}$; green down triangles), medium $\left(0.5\right.$ c.u. $<F_{150 \mathrm{GeV}}<1.0$ c.u.; blue open circles), or low $\left(F_{150 \mathrm{GeV}}<0.5\right.$ c.u.; pink open squares). Vertical bars denote $1 \sigma$ uncertainties, horizontal bars denote energy bins. Lines show best fits using log-parabolic (for flare nights) and power-law (for high/medium/low flux levels) functions. The insets report the spectral indices derived from the fit, as well as the goodness of such fit. The spectra are corrected for EBL extinction using [6]'s 'Low' EBL model. 